\title{
Estrategia de telesalud para mejorar el diagnóstico de EPOC y asma en México en el primer nivel de atención
}

\author{
E-health strategy to improve the diagnosis of COPD \\ and asthma in México's Primary Care
}

\author{
Juan Carlos Vázquez-García, ${ }^{*}$ Rosaura Esperanza Benítez-Pérez,* Elisa Sánchez-Gallén,* \\ Margarita Fernández-Vega, Rafael de Jesús Hernández-Zenteno,, Jorge Salas-Hernández,* \\ Rogelio Pérez-Padilla, ${ }^{*}$ Adela Reyes-Herrera, ${ }^{\ddagger}$ Diana Ruiz-Ascencio, ${ }^{\ddagger}$ Robert Camargo-Ángeles ${ }^{\ddagger}$
}

*Instituto Nacional de Enfermedades Respiratorias Ismael Cosío Villegas, Ciudad de México;

${ }^{\ddagger}$ Centro Nacional de Programas Preventivos y Control de Enfermedades.

Palabras clave: Telemedicina, enfermedad pulmonar obstructiva crónica, asma, atención primaria.

Keywords: Telemedicine, pulmonary disease chronic obstructive, asma, primary health care.

\section{LA CARGA DE LAS ENFERMEDADES PULMONARES OBSTRUCTIVAS: EPOC Y ASMA}

Las enfermedades respiratorias son causa frecuente de morbilidad y mortalidad en México y en el mundo. La contribución de las mismas a la carga mundial es muy relevante si se suman todas, con independencia del grupo de clasificación internacional en el que se encuentren. ${ }^{1}$ Clasificadas dentro del grupo de enfermedades no transmisibles por la Organización Mundial de la Salud (OMS), las patologías respiratorias ocasionan alrededor de 3.9 millones de muertes anuales. ${ }^{2}$

Para 2016 había más de 336 millones de personas con asma en el mundo. En ese mismo año, el asma causó 417,918 fallecimientos en el ámbito mundial y provocó la

Correspondencia:

Dra. Rosaura Esperanza Benítez Pérez

Instituto Nacional de Enfermedades Respiratorias Ismael Cosío

Villegas, Ciudad de México.

Correo electrónico: drarebp@gmail.com

Recibido: 30-X-2020; aceptado: 17-XI-2020.

Citar como: Vázquez-García JC, Benítez-Pérez RE, Sánchez-Gallén E, Fernández-Vega M, Hernández-Zenteno RJ, Salas-Hernández J, et al. Estrategia de telesalud para mejorar el diagnóstico de EPOC y asma en México en el primer nivel de atención. Neumol Cir Torax. 2021; 80 (1): 11-18. https:// dx.doi.org/10.35366/99449 pérdida de 24.8 millones de años de vida, ajustados en función de la discapacidad. ${ }^{3}$

De acuerdo con el estudio de carga de la morbilidad, en 2016 se registraron 251 millones de casos de enfermedad pulmonar obstructiva crónica (EPOC), estimándose 3.17 millones de fallecimientos por esta causa en el mismo año. ${ }^{4}$ El accidente cerebrovascular y la enfermedad pulmonar obstructiva crónica son la segunda y tercera causas de defunción en el mundo, que representan aproximadamente el 11 y $6 \%$ del total de muertes, respectivamente. ${ }^{5}$

En México, durante 2018 se informó que dentro de las primeras diez causas de muerte se sitúa la EPOC, con 26, 618 fallecimientos con una tasa de mortalidad de 21.55 por 100 mil habitantes. ${ }^{6}$

En referencia al asma para el año 2019, en México se registraron 254,713 casos, con una tasa de incidencia de 201.23 por 100 mil habitantes, ocupando el lugar 13 de morbilidad. ${ }^{6}$ En el año 2018 se registraron 1,306 fallecimientos, que representa una tasa de 1.06 por 100 mil habitantes. ${ }^{6}$ Este tipo de fallecimientos se consideran en su mayoría totalmente prevenibles.

El estudio PLATINO, ${ }^{7}$ con base poblacional realizado en el Área Metropolitana de la Ciudad de México en sujetos de 40 años o más mostró una prevalencia de EPOC del 7.8\%, diagnóstico hecho con el estándar de oro actual, la espirometría. Asimismo, varias encuestas nacionales, principalmente utilizando el cuestionario estandarizado de ISAAC (del inglés, International Survey for Allergies And Asthma in Childhood) muestran una prevalencia de asma, diagnosticada previamente por médicos de $4-10 \%$ de la población. ${ }^{8-18}$ Sin embargo, si se define por sibilancias en el último año, la prevalencia 
Neumol Cir Torax. 2021; 80 (1): 11-18

es de aproximadamente el doble. El asma es la enfermedad pulmonar más frecuente y la padecen cerca de 8.5 millones de mexicanos. ${ }^{19}$

La morbilidad y los gastos que se derivan de las enfermedades respiratorias son enormes. Por ejemplo, recién se estimó por el Instituto Nacional de Salud Pública que el costo anual de las enfermedades relacionadas al tabaquismo es de $\$ 45$ mil millones de pesos, y corresponden a 8-15\% del gasto en salud, siendo inclusive una subestimación ya que sólo se investigaron cuatro padecimientos: EPOC, cáncer pulmonar, enfermedad isquémica miocárdica y enfermedad cerebrovascular. ${ }^{20}$ En el Instituto Nacional de Enfermedades Respiratorias Ismael Cosío Villegas (INER), Ciudad de México, el gasto por EPOC fue de 52 millones de pesos por 508 pacientes, un promedio de $\$ 102,362$ por paciente y los gastos de bolsillo son proporcionales a la gravedad de la enfermedad. En el año 2014 se estimaron de $\$ 1,116$ a $\$ 2,240$ USD por año. En los Estados Unidos, para 13.7 millones de personas con EPOC se ha estimado un costo anual total de 36 mil millones de dólares. ${ }^{21}$

\section{EL GRAN PROBLEMA DEL SUBDIAGNÓSTICO Y ERROR DIAGNÓSTICO DE EPOC Y ASMA}

En el estudio PLATINO se demostró que el $86 \%$ de las personas con EPOC no habían sido diagnosticadas (subdiagnóstico); además, se encontró que aproximadamente la mitad de los individuos con diagnóstico previo de EPOC, bronquitis crónica o enfisema nunca se habían realizado una espirometría (error diagnóstico). ${ }^{22}$ La razón principal de este hecho se debe a que el diagnóstico de EPOC requiere ser confirmado por medio de espirometría, un método objetivo que demuestra la obstrucción al flujo del aire. ${ }^{23}$ De forma similar, el diagnóstico de asma se establece con la confirmación de obstrucción variable al flujo de aire por medio de la espirometría. 19,24

La espirometría es una prueba equivalente a la medición de la presión arterial para el diagnóstico de hipertensión o a la medición de la glucosa en sangre para el diagnóstico de diabetes. Sin embargo, se realiza muy pobremente en nuestro medio, en especial en centros de atención médica primaria y secundaria, por lo que es indispensable que se incremente su uso en sujetos de alto riesgo o probabilidad de padecer la enfermedad. El subdiagnóstico y el error diagnóstico son problemas mayores de EPOC y asma, principalmente en medios de atención primaria y secundaria. Esto es dependiente en gran medida del acceso a la prueba de espirometría, un reto mayor constituido por su implementación y uso adecuado. ${ }^{25}$ En México y en la mayoría de los países en desarrollo se utiliza de manera muy pobre, a pesar de su relevancia diagnóstica y de los esfuerzos por actualizar guías para su uso en el consultorio. ${ }^{26-28}$ Recientemente se publicó que 44 ciudades pertenecientes a 27 países presentan un subdiagnóstico poblacional de EPOC del 81.4\% (50-98\%); este estudio incluyó nueve ciudades de América Latina donde la falta de diagnóstico fue del 77 al 95\%, incluyendo la Ciudad de México con $81.6 \% .{ }^{29}$ En Estados Unidos, la prevalencia de neumopatía obstructiva no diagnosticada rebasa el $70 \%$ y se ha mantenido prácticamente sin cambio en los últimos 20 años. ${ }^{30}$

A diferencia de los centros de referencia que concentran los casos de alto riesgo y los casos graves ya diagnosticados, los centros de atención primaria y secundaria serían los principales sitios para establecer estrategias que favorezcan la detección y el diagnóstico de EPOC y asma, sobre todo en etapas tempranas. En un estudio canadiense de 1,003 pacientes mayores de 40 años reclutados de centros de medicina familiar y con historia de tabaquismo mayor a 20 paquetes/año, se encontró una prevalencia de EPOC del 20.7\%; el subdiagnóstico de EPOC fue del $68 \%$ mientras que en otros 43 pacientes (4.3\%) con diagnóstico previo de EPOC no se confirmó la obstrucción al flujo aéreo (error diagnóstico). ${ }^{31}$ En un estudio similar de cuatro países de América Latina (Argentina, Colombia, Venezuela y Uruguay), se reclutaron 1,540 pacientes mayores de 40 años con alto riesgo de padecer EPOC, quienes completaron espirometría posbroncodilatador; la prevalencia de EPOC subdiagnosticado fue del $77 \%$ y la de error diagnóstico del $30.4 \%{ }^{32}$ En México, Laniado-Laborin et al describieron a 2,297 pacientes mayores de 40 años con alto riesgo de padecer EPOC, quienes completaron evaluación y espirometría posbroncodilatador en centros de atención primaria de 27 ciudades de 20 estados de la República. La prevalencia de EPOC fue de $20.6 \%$ (GOLD I-IV) y de $6.9 \%$ para EPOC grave o muy grave (GOLD III y IV). ${ }^{33}$ Esta experiencia nacional e internacional demuestra cómo la espirometría diagnóstica realizada en pacientes de alto riesgo y medios de atención primaria puede ser altamente eficiente en la detección de EPOC. Además, la evidencia actual no es lo suficientemente contundente para recomendar el tamizaje de todos los sujetos asintomáticos con factores de riesgo para esta enfermedad. . $^{34,35}$

A pesar de que el asma es la enfermedad pulmonar crónica más frecuente, exhibe un escenario de mala detección y error diagnóstico frecuente, así como tratamiento deficiente y baja cobertura médica. ${ }^{36-38}$ Una recomendación general para el diagnóstico es no basarlo de manera exclusiva en síntomas y demostrar la obstrucción al flujo de aire, una condición que hace necesaria la espirometría. 19,24 En países desarrollados, en el nivel primario de atención se ha descrito que el subdiagnóstico de asma puede ser superior al $40 \%$ mientras que el error diagnóstico supera el $30 \% .{ }^{39}$ Esto también sustenta la necesidad de implementar programas de espirometría para confirmar el diagnóstico; además, deben impactar en un mejor manejo y ajuste de tratamiento. ${ }^{40-42}$ 


\section{IMPLEMENTACIÓN DE LA ESPIROMETRÍA EN EL PRIMER Y SEGUNDO NIVEL DE ATENCIÓN}

La espirometría forzada es una prueba de función respiratoria de tipo ventilatorio que se realiza en menos de 15 minutos; requiere de una maniobra de soplar que se realiza con un dispositivo (espirómetro) que mide cronométricamente en segundos el máximo volumen o flujo de aire que se puede exhalar con el mayor esfuerzo y después de una inspiración máxima. Al mayor volumen de aire medido en litros, que se puede exhalar durante la maniobra, se le llama capacidad vital forzada (FVC) mientras que el volumen que se exhala durante el primer segundo se le conoce como volumen espiratorio forzado en un segundo $\left(\mathrm{FEV}_{1}\right)$. Una prueba de calidad requiere de tres maniobras que cumplan los criterios de aceptabilidad establecidos y una máxima coincidencia (repetibilidad) entre los mayores valores tanto para FVC como FEV $1(<0.15 \mathrm{~L}) .{ }^{43,44}$ Una persona adulta con pulmones sanos debe exhalar aproximadamente más del $70 \%$ de su FVC en el primer segundo (relación o cociente $\mathrm{FEV}_{1} / \mathrm{FVC}$ ). La FVC es un parámetro representativo del volumen total o tamaño de los pulmones y el cociente $\mathrm{FEV}_{1} / \mathrm{FVC}$ define la presencia o no de obstrucción al flujo de aire. Un cociente $\mathrm{FEV}_{1} / \mathrm{FVC}$ menor a la percentil $5 \mathrm{o}$ límite inferior normal para la edad, sexo y estatura de una persona es diagnóstico de obstrucción al flujo aéreo, y la espirometría es el estándar de oro para este diagnóstico. ${ }^{45}$ La espirometría diagnóstica debe incluir una prueba de respuesta al broncodilatador. Posterior a una espirometría basal se administran de 200 a 400 microgramos de salbutamol en aerosol (inhalador de dosis medida) y 15 minutos después se repite la prueba. Una mejoría mayor a 0.20 L y $12 \%$ en el volumen de FVC y/o FEV ${ }_{1}$ define una respuesta positiva al broncodilatador. ${ }^{44}$ La presencia de obstrucción al flujo de aire con respuesta positiva al broncodilatador, particularmente cuando revierte la obstrucción, sugiere el diagnóstico de asma, mientras que la obstrucción persistente posterior al broncodilatador es compatible con el diagnóstico de EPOC.

Además de la inversión en infraestructura tecnológica y de recursos humanos, el mayor reto en la implementación de la espirometría es asegurar que la prueba se realice bajo los mejores estándares de calidad para que resulte confiable para el diagnóstico de EPOC y asma. Los requerimientos indispensables para una prueba confiable son contar con un equipo exacto y preciso, así como la capacitación y vigilancia del personal que ejecuta la prueba. ${ }^{46} \mathrm{Si}$ bien la espirometría se puede realizar casi en cualquier ambiente y circunstancia, como el consultorio o domicilio, con frecuencia puede no ser confiable por ser de mala calidad. ${ }^{47}$ La meta más importante al implementar e interpretar la espirometría es minimizar la tasa de interpretación errónea a causa de la mala calidad de las pruebas y a la falta de entrenamiento y vigilancia del personal que la realiza. ${ }^{48,49}$ En estudios de investigación multinacional, la calidad de las espirometrías realizadas por personal previamente capacitado con cursos estandarizados de sólo dos días de duración, seguidos con un programa de supervisión, puede superar el $90 \%$ de pruebas bajo estándares internacionales. ${ }^{50}$

Es muy importante que aquéllos que realizan espirometrías en medios de atención primaria se encuentren entrenados y acreditados, que sean capaces de demostrar su competencia en la ejecución correcta de las pruebas, la identificación de errores y la interpretación apropiada de los resultados. Además, se recomienda establecer un programa de control de calidad destinado a la vigilancia, tanto del personal de salud ya certificado como de la estabilidad de los equipos utilizados en el consultorio. ${ }^{48,51} \mathrm{Sin}$ embargo, la calidad informada de las pruebas realizadas en centros primarios es muy variable. ${ }^{52-55}$ Se han implementado diversas estrategias para obtener espirometrías de buena calidad en los consultorios, con resultados mixtos. ${ }^{56}$ Las más prometedoras han involucrado programas de monitoreo a distancia, usando tecnologías de comunicación (telemedicina), equipos de diagnóstico itinerantes que visitan periódicamente los centros de atención primaria o programas de entrenamiento y seguimiento estrecho. ${ }^{56-59}$ Estos modelos han informado una proporción de estudios con calidad que alcanza más del $80 \%$, aunque hasta el momento no hay algún estudio que demuestre la superioridad de estas propuestas.

\section{TELE-ESPIROMETRÍA Y RED NACIONAL DE ESPIROMETRÍA (RENACE)}

La tecnología actual y la experiencia previa ganada en el INER, principalmente gracias a sus modelos educativos y grandes estudios multicéntricos de investigación, han demostrado que es posible realizar la prueba a distancia (fuera de un laboratorio de fisiología pulmonar) y con la mayor calidad. ${ }^{60,61}$ Pero para la implementación de la prueba en centros primarios y secundarios de todo el país, se requiere del desarrollo de una estrategia especial que garantice eficiencia en la capacitación, el seguimiento y los resultados de la prueba con fines de detección y adecuado diagnóstico de los enfermos. Un modelo de telemedicina implica el uso de tecnologías de informática y comunicación para ofrecer un servicio médico a distancia, en especial cuando existe un acceso difícil a los servicios especializados. ${ }^{62}$

Dadas las características geográficas del país y la disparidad en el acceso a recursos médicos de especialidad, se decidió implementar un modelo de telediagnóstico de EPOC y asma basado en tele-espirometría. Este modelo consiste en la implementación de centros de diagnóstico espirométrico en unidades de atención primaria y hospitales de segundo nivel que son seleccionados de todo el país 
para ser incluidos, capacitados, certificados y enlazados con una permanente comunicación y monitoreo desde una Unidad de Telemedicina del INER (UTM-INER), la cual vigila la calidad de las pruebas y realiza una interpretación. A este programa le hemos Ilamado RENACE (Red Nacional de Espirometría). Las estrategias específicas definidas para la implementación del programa se describen en la Figura 1.

Evaluación inicial: desde finales del año 2015 se ha establecido un esquema de trabajo conjunto de análisis entre el programa que coordina la parte normativa en la Secretaría de Salud (SSA) y el INER para establecer un diagnóstico situacional con respecto a la infraestructura con que se cuenta en cada centro de diagnóstico que sea candidato potencial. Este diagnóstico se refiere a espacios físicos, tecnológicos y de comunicación, así como recursos humanos y financieros disponibles para la implementación del programa.

Selección de equipos: la adecuada selección de equipos de espirometría es fundamental; el equipo elegido debe ser preciso y exacto, con buen desempeño técnico y que permita facilidades de conectividad y comunicación. Es deseable que pueda personalizarse con fines de captura y manejo de la información a distancia. Asimismo, es recomendable que los costos de inversión y de operación sean razonables y asequibles.

Selección de los centros: la selección de los centros de salud y hospitales provenientes fue realizada por las 32 entidades federativas. El criterio de selección fue usado en la localización estratégica para la referencia de los pacientes, la realización de las pruebas, la infraestructura del centro, así como la participación y eficiencia demostrada en otros programas, principalmente en el programa nacional de tuberculosis.

Selección del personal: la cuidadosa selección del personal para ser capacitado e integrado al programa es un paso clave para la optimización de recursos y tiempo para la implementación del programa. El personal seleccionado puede incluir médicos, enfermeras y técnicos que muestren interés y entusiasmo, así como factibilidad de desempeño laboral de largo plazo en el centro de referencia.
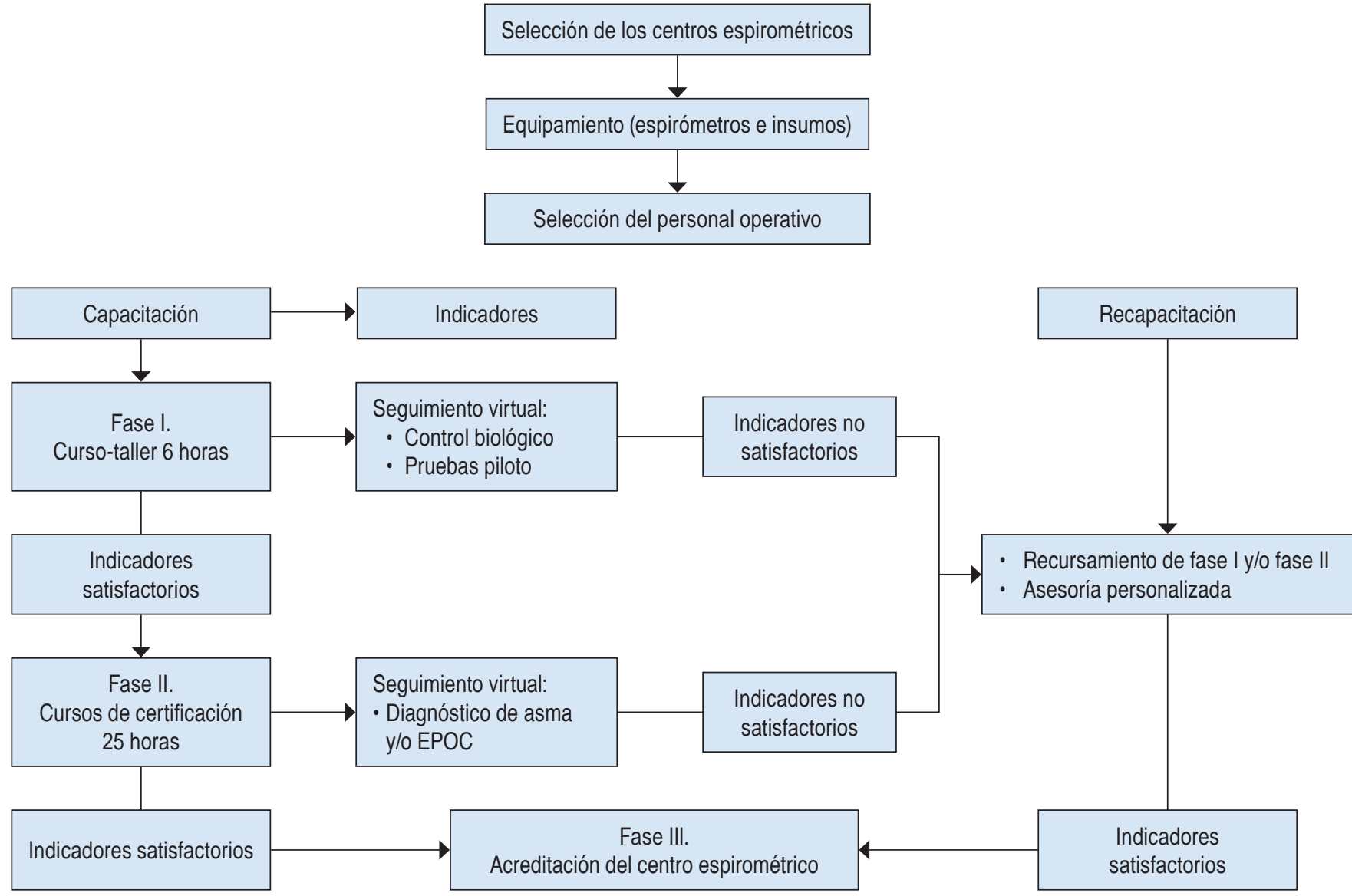

Figura 1: Diagrama de flujo para la selección de centros de espirometría y del personal, así como proceso de capacitación, seguimiento y certificación final del centro.

$\mathrm{EPOC}=$ enfermedad pulmonar obstructiva crónica. 
Capacitación inicial en espirometría (fase I): todo el personal seleccionado deberá completar una capacitación inicial enfocada en la realización de espirometría «aprendiendo a hacer espirometría», que consiste en un curso teórico en línea de cuatro a seis horas de duración, así como práctico presencial de cuatro horas de duración que tiene como objetivos de aprendizaje adquirir los conceptos básicos de tipo fisiológico, técnico y clínico, así como el entrenamiento básico para el uso del espirómetro, la realización de la prueba y el proceso de comunicación inicial con la UTM-INER.

Implementación y seguimiento inicial: posterior a la capacitación inicial, cada centro debe implementar la prueba y realizar un control de calidad inicial de tipo biológico, que consiste en la realización semanal de una espirometría autorrealizada por el mismo técnico o por alguien más del centro que reúna las características requeridas. Asimismo, deben realizar pruebas piloto y a pacientes reales para establecer una experiencia local inicial, así como la comunicación y el monitoreo desde la UTM-INER para la interpretación y retroalimentación al centro. Esta etapa tiene una duración no menor a tres meses.

Certificación de técnicos en espirometría (fase II): todos los técnicos y centros de diagnóstico espirométrico que cumplan satisfactoriamente las fases I y II podrán completar una segunda capacitación teórico-práctico y de certificación en espirometría bajo los estándares internacionales: curso de espirometría INER. ${ }^{60}$ Este es un curso mixto (en línea y presencial) de 25 horas de duración, donde se revisan las bases fisiológicas de la respiración, la descripción amplia de los espirómetros, su control de calidad, los procedimientos de realización de la prueba simple y con broncodilatador y la interpretación de la misma. El curso cuenta con los talleres suficientes para que los alumnos adquieran el entrenamiento y la competencia suficiente para la realización de la prueba bajo los estándares internacionales para que al final presenten una evaluación teórica y práctica con fines de certificación. El curso requiere el $100 \%$ de asistencia y una aprobación de más del 70\% de las evaluaciones.

Seguimiento y monitoreo: posterior a la certificación de los técnicos en espirometría, éstos y sus centros deberán completar un nuevo seguimiento por espacio de hasta seis meses para la vigilancia de los controles de calidad, graduación de la calidad de las pruebas e interpretación centralizada desde el INER en cuanto al diagnóstico espirométrico del patrón ventilatorio (normal, obstructivo o sugestivo de restricción), así como el diagnóstico clínico de EPOC y asma, si se cuenta con la información suficiente.

Certificación del centro (fase III): los centros que demuestren trabajo regular con controles de calidad y una proporción suficientemente alta de pruebas de buena calidad serán certificados por el INER y la Sociedad Mexicana de Neumología y Cirugía de Tórax (SMNyCT) como Centros de Diagnóstico Espirométrico. Esta certificación tendrá duración de un año y al término del mismo será renovada de forma automática, si el centro mantiene los estándares de calidad requeridos.

Indicadores de calidad y eficiencia: cada centro de diagnóstico, los estados y el programa RENACE, en lo general, serán monitoreados y evaluados de manera permanente de acuerdo con indicadores específicos de eficiencia y calidad que serán informados de forma oportuna y transparente. Los indicadores del programa que pueden medirse de manera objetiva serán los siguientes:

1. Número de centros incluidos en el programa.

2. Número de personas entrenadas y certificadas para la realización y supervisión de las pruebas de espirometría.

3. Número de centros certificados.

4. Cobertura estatal y nacional de Centros de Diagnóstico Espirométrico.

5. Número total de pacientes referidos a espirometría.

6. Número de espirometrías realizadas.

7. Número de espirometrías de adecuada calidad para interpretación.

8. Número de espirometrías con patrones ventilatorios anormales.

9. Número de espirometrías con diagnóstico de obstrucción al flujo de aire.

10. Número de pacientes con diagnóstico establecido de asma o EPOC.

\section{IMPLEMENTACIÓN: ALCANCES, PROBLEMÁTICAS Y PERSPECTIVAS FUTURAS}

Antes del inicio del programa (2015), se realizó un censo en las unidades de primer y segundo nivel de atención, identificando únicamente 15 espirómetros en hospitales y ningún espirómetro funcional en los centros de atención primaria. Al momento de la publicación de este artículo, se ha alcanzado la cobertura en equipamiento e insumos (espirómetros y fármacos) en los 32 estados de la República Mexicana, con un total de 250 espirómetros. De igual manera, se ha alcanzado la capacitación en fase I de 672 personas procedentes de 31 estados, en fase II de 254 personas y 25 centros espirométricos certificados. Sin embargo, la continuidad del personal inicialmente capacitado en el programa es multifactorial y desafortunadamente sólo el $13 \%$ de los inicialmente capacitados en fase I terminan el proceso. La pandemia de COVID-19 llevó a una desaceleración en el proceso de capacitación y un cese temporal en la ejecución de los estudios espirométricos; no obstante, también ha sido 
un momento de oportunidad de plantear y desarrollar estrategias alternativas de capacitación y recapacitación con involucro mayor de las tecnologías de la información mediante el desarrollo de cursos totalmente en línea y asesoría a distancia con un modelo «train the trainer».

La implementación total del programa en la República Mexicana implica compromiso e inversión en la que deben participar, tanto el sector público como el privado buscando como objetivo final mejorar la detección, diagnóstico y seguimiento de los enfermos con EPOC y asma.

\section{Agradecimientos}

Este proyecto fue implementado gracias al apoyo de diferentes grupos de interés comprometidos con el fortalecimiento de la atención de la salud respiratoria, entre ellos AstraZeneca.

\section{REFERENCIAS}

1. Perez-Padilla R. Hidden respiratory disease-associated deaths. Int J Tuberc Lung Dis. 2008;12(4):458-464.

2. WHO. [Fecha de consulta: 30 de octubre de 2020]. Accesible en: https:// www.who.int/en/news-room/fact-sheets/detail/noncommunicablediseases.

3. WHO. [Fecha de consulta: 27 de octubre de 2020]. Accesible en: https://www.who.int/es/news-room/fact-sheets/detail/asthma.

4. WHO. [Fecha de consulta: 27 de octubre de 2020]. Accesible en: https://www.who.int/news-room/fact-sheets/detail/chronicobstructive-pulmonary-disease-(copd).

5. WHO. [Fecha de consulta: 27 de octubre de 2020]. Accesible en: https://www.who.int/es/news-room/fact-sheets/detail/the-top-10causes-of-death.

6. Cubos de mortalidad/DGIS/SS/2020. [Fecha de consulta: 27 de octubre de 2020]. Accesible en: http://www.dgis.salud.gob.mx/ contenidos/basesdedatos/bdc_defunciones_gobmx.html.

7. Menezes AMB, Perez-Padilla R, Jardim JRB, Muiño A, Lopez MV, Valdivia G, et al.; PLATINO Team. Chronic obstructive pulmonary disease in five Latin American cities (the PLATINO study): a prevalence study. Lancet. 2005;366(9500):1875-1881. https://doi. org/10.1016/s0140-6736(05)67632-5.

8. Piedras MMP, Huerta LJ. Mortalidad por asma. Alerg Asma Inmunol Pediatr. 2011;20(3):107-119.

9. Del-Rio-Navarro B, Del Rio-Chivardi JM, Berber A, Sienra-Monge JJL, Rosas-Vargas MA, Baeza-Bacab M. Asthma prevalence in children living in north Mexico City and a comparison with other Latin American cities and world regions. Allergy Asthma Proc. 2006;27(4):334-340. https://doi.org/10.2500/aap.2006.27.2880.

10. Del-Rio-Navarro B, Berber A, Blandón-Vijil V, Ramírez-Aguilar M, Romieu I, Ramírez-Chanona N, et al. Identification of asthma risk factors in Mexico City in an International Study of Asthma and Allergy in Childhood survey. Allergy Asthma Proc. 2006;27(4):325-333. https:// doi.org/10.2500/aap.2006.27.2874

11. Morfín-Maciel B, Barragán-Meijueiro M de L, Nava-Ocampo AA. Individual and family household smoking habits as risk factors for wheezing among adolescents. Prev Med. 2006;43(2):98-100. https:// doi.org/10.1016/j.ypmed.2006.04.002.
12. Violante R, del Río Navarro BE, Berber A, Ramírez Chanona N, Baeza Bacab M, Sienra Monge JJ. Obesity risk factors in thE ISAAC (International Study of Asthma and Allergies in Childhood) in Mexico City. Rev Alerg Mex. 2005;52(4):141-145.

13. Del Río-Navarro BE, Hernández-Román MP, Espinola Reyna G, Berber A, Escalante-Dominguez AJ, González-Reyes M, et al. A comparative study of bronchodilator reversibility with albuterol, between asthma symptomatic and asymptomatic children according to ISAAC questionnaire in Mexico City. Allergol Immunopathol (Madr). 2004;32(6):334-339. https://doi.org/10.1016/s0301-0546(04)79265-4.

14. Barraza-Villarreal A, Sanín-Aguirre LH, Téllez-Rojo MM, LacasañaNavarro M, Romieu I. Prevalence of asthma and other allergic diseases in school children from Juarez City, Chihuahua. Salud Publica Mex. 2001;43(5):433-443.

15. Mendoza-Mendoza A, Romero-Cancio JA, Pena-Rios HD, Vargas $\mathrm{MH}$. Prevalence of asthma in schoolchildren from the Mexican City Hermosillo. Gac Med Mex. 2001;137(5):397-401.

16. Rojas Molina N, Legorreta Soberanis J, Olvera Guerra F. Prevalence and asthma risk factors in municipalities of the State of Guerrero, Mexico. Rev Alerg Mex. 2001;48(4):115-118.

17. Tatto-Cano MI, Sanín-Aguirre LH, González V, Ruiz-Velasco S, Romieu I. Prevalence of asthma, rhinitis and eczema in school children in the City of Cuernavaca, Mexico. Salud Publica Mex. 1997;39(6):497-506.

18. García-Sancho C, Fernández-Plata R, Martínez-Briseño D, FrancoMarina F, Pérez-Padilla JR. Prevalencia y riesgos asociados con pacientes adultos con asma de 40 años o más de la Ciudad de México: estudio de base poblacional. Salud Publica Mex. 2012;54(4):425432. Accesible en: https://saludpublica.mx/index.php/spm/article/ view/7156.

19. Guía Mexicana de Asma. Neumol Cir Torax. 2017;76(suppl):s1-s136.

20. Reynales-Shigematsu LM. Costos de atención médica de las enfermedades atribuibles al consumo de tabaco en América: revisión de la literatura. Salud Publica Mex. 2006;48(suppl I):S190-S200.

21. Fernández-Plata R, Martínez-Briseño D, García-Sancho F, CanoJiménez D, Ramírez-Venegas A, Sansores-Martínez R, et al. Métodos para la estimación de costos en salud de la EPOC: resultados basales. Neumol Cir Torax. 2016;75(1):4-11.

22. Tálamo C, de Oca MM, Halbert R, Pérez-Padilla R, Jardim JRB, Muiño A, et al.; PLATINO team. Diagnostic labeling of COPD in five Latin American cities. Chest. 2007;131(1):60-67. https://doi.org/10.1378/ chest.06-1149.

23. Guía de Práctica Clínica Mexicana para el diagnóstico y tratamiento de la Enfermedad Pulmonar Obstructiva Crónica. Neumol Cir Torax. 2019;78(suppl):s20-s22.

24. Global Strategy for Asthma Management and Prevention, Global Initiative for Asthma (GINA) 2017 Update. Available from: http:// ginasthma.org/.

25. The Royal College of Physicians of London/BTS/BLF. Report 5 of The National Chronic Obstructive Pulmonary Disease Audit 2008: survey of COPD care within UK General Practices. 2008. [Accessed October 27, 2020]. Available at https://www.rcplondon.ac.uk/projects/ outputs/national-copd-audit-2008.

26. Levy ML, Quanjer PH, Booker R, Cooper BG, Holmers S, Small I; General Practice Airways Group. Diagnostic spirometry in primary care. Proposed standards for general practice compliant with American Thoracic Society and European Respiratory Society recommendations. Prim Care Respir J. 2009;18(3):130-147. https:// doi.org/10.4104/pcrj.2009.00054. 
Neumol Cir Torax. 2021; 80 (1): 11-18

27. Coates AL, Graham BL, McFadden RG, McParland C, Moosa D, Provencher S, et al. Spirometry in primary care. Can Respir J. 2013;20(1):13-21. https://doi.org/10.1155/2013/615281.

28. Ferguson GT, Enright PL, Buist AS, Higgins MW. Office spirometry for lung health assessment in adults: a consensus statement from the National Lung Health Education Program. Respir Care. 2000;45(5):513-530.

29. Lamprecht B, Soriano JB, Studnicka M, Kaiser B, Vanfleteren LE, Gnatiuc $L$, et al. Determinants of underdiagnosis of COPD in national and international surveys. Chest. 2015;148(4):971-985. https://doi. org/10.1378/chest.14-2535.

30. Martinez CH, Mannino DM, Jaimes FA, Curtis JL, Han MK, Hansel NN, et al. Undiagnosed obstructive lung disease in the United States. Associated Factors and long-term mortality. Ann Am Thoracic Soc. 2015;12(12):1788-1795. doi: 10.1513/AnnalsATS.201506-388OC.

31. Hill K, Goldstein RS, Guyatt GH, Blouin M, Tan WC, Davis LL, et al. Prevalence and underdiagnosis of chronic obstructive pulmonary disease among patients at risk in primary care. CMAJ. 2010;182(7):673-678. https://doi.org/10.1503/cmaj.091784.

32. Casas HA, Montes OM, López VM, Aguirre C, Schiavi E, Jardim JR; PUMA Team. COPD underdiagnosis and misdiagnosis in a high-risk primary care population in four Latin American countries. A key to enhance disease diagnosis: The PUMA Study. PLoS One. 2016;11(4):e0152266. https://doi.org/10.1371/journal.pone.0152266.

33. Laniado-Laborin R, Rendón A, Bauerle O. Chronic obstructive pulmonary disease case finding in Mexico in an at-risk population. Int J Tuberc Lung Dis. 2011;15(6):818-823. https://doi.org/10.5588/ ijtld.10.0546.

34. Sansores RH, Velázquez-Uncal M, Pérez-Bautista O, Villalba-Caloca J, Farfán-Valencia R, Ramírez-Venegas A. Prevalence of chronic obstructive pulmonary disease in asymptomatic smokers. Int J Chron Obstruct Pulmon Dis. 2015;10:2357-2363. https://doi.org/10.2147/ copd.s91742.

35. Guirguis-Blake JM, Senger CA, Webber EM, Mularski R, Whitlock EP. Screening for chronic obstructive pulmonary disease: A systematic evidence review for the U.S. preventive services task force [Internet]. Rockville (MD): Agency for Healthcare Research and Quality (US); 2016 Apr. (Evidence Syntheses, No. 130.) Available from: https:// www.ncbi.nlm.nih.gov/books/NBK361185/.

36. Aaron SD, Vandemheen KL, FitzGerald JM, Ainslie M, Gupta S, Lemière $\mathrm{C}$, et al. Reevaluation of diagnosis in adults with physiciandiagnosed asthma. JAMA. 2017;317(3):269-279. https://doi. org/10.1001/jama.2016.19627.

37. Looijmans-Van den Akker I, van Luijn K, Verheij T. Overdiagnosis of asthma in children in primary care: a retrospective analysis. Br J Gen Pract. 2016;66(644):e152-e157. https://doi.org/10.3399/ bjgp16x683965.

38. José BP, Camargos PA, Cruz Filho AA, Correa R de A. Diagnostic accuracy of respiratory diseases in primary health units. Rev Assoc Med Bras (1992). 2014;60(6):599-612. https://doi.org/10.1590/18069282.60.06.021.

39. MacNeil J, Loves RH, Aaron SD. Addressing the misdiagnosis of asthma in adults: where does it go wrong? Expert Rev Respir Med. 2016;10(11):1187-1198. https://doi.org/10.1080/17476348.2016.1242415.

40. Haahtela T, Tuomisto LE, Pietinalho A, Klaukka T, Erhola M, Kaila $M$, et al. A 10 year asthma programme in Finland: Major change for the better. Thorax. 2006;61(8):663-670. https://doi.org/10.1136/ thx.2005.055699.
41. Tuomisto L, Erhola M, Kaila M, Brander PE, Puolijoki H, Kauppinen $\mathrm{R}$, et al. Asthma Programme in Finland: high consensus between general practitioners and pulmonologists on the contents of an asthma referral letter. Prim Care Respir J. 2004;13(4):205-210. https://doi. org/10.1016/j.pcrj.2004.04.001.

42. Tuomisto LE, Erhola M, Kaila M, Brander PE, Kauppinen R, Puolijoki $\mathrm{H}$, et al. The Finnish national asthma programme: Communication in asthma care-quality assessment of asthma referral letters. J Eval Clin Pract. 2007;13(1):50-54. https://doi.org/10.1111/j.13652753.2006.00645.x.

43. Miller MR, Hankinson J, Brusasco V, Burgos F, Casaburi R, Coates A, et al. Standardisation of spirometry. Eur Respir J. 2005;26(2):319338. https://doi.org/10.1183/09031936.05.00034805.

44. Graham BL, Steenbruggen I, Miller MR, Barjaktarevic IZ, Cooper BG, Hall GL, et al. Standardization of spirometry 2019 update. An Official American Thoracic Society and European Respiratory Society Technical Statement. Guideline 2019. https://doi.org/10.1164/ rccm.201908-1590ST.

45. Pellegrino R, Viegi G, Brusasco V, Crapo RO, Burgos F, Casaburi $\mathrm{R}$, et al. Interpretative strategies for lung function tests. Eur Respir J. 2005;26(5):948-968. https://doi.org/10.1183/09031936.05.00035205.

46. Pérez-Padilla R, Vázquez-García JC, Márquez MN, Menezes AMB; PLATINO Group. Spirometry quality-control strategies in a multinational study of the prevalence of chronic obstructive pulmonary disease. Respir Care. 2008;53(8):1019-1026.

47. Enright $P$, Schermer $T$. Don't pay for poor quality spirometry tests. Prim Care Respir J. 2013;22(1):15-16. https://doi.org/10.4104/ pcrj.2013.00023.

48. Perez-Padilla R, Torre Bouscoulet L, Vázquez García JC. Implementing a spirometry program. Int J Tuberc Lung Dis. 2016;20(9):1142. https:// doi.org/10.5588/ijtld.16.0512.

49. Eaton T, Withy S, Garrett JE, Mercer J, Whitlock RM, Rea HH Spirometry in primary care practice: the importance of quality assurance and the impact of spirometry workshops. Chest. 1999;116(2):416-423. https://doi.org/10.1378/chest.116.2.416.

50. Enright $P$, Vollmer WM, Lamprecht B, Jensen R, Jithoo A, Tan W, et al. Quality of spirometry tests performed by 9893 adults in 14 countries: the BOLD Study. Respir Med. 2011;105(10):1507-1515. https://doi. org/10.1016/j.rmed.2011.04.008.

51. Enright PL. Should we keep pushing for a spirometer in every doctor's office? Respir Care. 2012;57(1):146-153. https://doi.org/10.4187/ respcare.01504.

52. Cawley MJ, Moon J, Reinhold J, Willey VJ, Warning li WJ. Spirometry: tool for pharmacy practitioners to expand direct patient care services. J Am Pharm Assoc (2003). 2013;53(3):307-315. https://doi. org/10.1331/japha.2013.12134.

53. Tuomisto L, Jarvinen V, Laitinen J, Erhola M, Kaila M, Brander P. Asthma Programme in Finland: the quality of primary care spirometry is good. Prim Care Respir J. 2008;17(4):226-231. https:// doi.org/10.3132/pcrj.2008.00053.

54. Licskai CJ, Sands TW, Paolatto L, Nicoletti I, Ferrone M. Spirometry in primary care: An analysis of spirometry test quality in a regional primary care asthma program. Can Respir J. 2012;19(4):249-254. https://doi.org/10.1155/2012/653084.

55. Johnston JC, Rempel C, Sanders C, Piggott E, Maxwell Y, Jaipersaud $\mathrm{K}$, et al. Introduction of spirometry into clinical practice in Georgetown, Guyana: quality and diagnostic outcomes. Int J Tuberc Lung Dis. 2016;20(9):1270-1274. https://doi.org/10.5588/ijtld.16.0068. 
56. Walters JA, Hansen EC, Johns DP, Blizzard EL, Walters EH, WoodBaker R. A mixed methods study to compare models of spirometry delivery in primary care for patients at risk of COPD. Thorax. 2008;63(5):408-414. https://doi.org/10.1136/thx.2007.082859.

57. Poels PJ, Schermer TR, Schellekens DP, Akkermans RP, de Vries Robbé PF, Kaplan A, et al. Impact of a spirometry expert system on general practitioners' decision making. Eur Respir J. 2008;31(1):8492. https://doi.org/10.1183/09031936.00012007.

58. Burgos F, Disdier C, de Santamaria EL, Galdiz B, Roger N, Rivera $\mathrm{ML}$, et al. Telemedicine enhances quality of forced spirometry in primary care. Eur Respir J. 2012;39(6):1313-1318. https://doi. org/10.1183/09031936.00168010.

59. Burgos F. Telemedicina en el laboratorio de función pulmonar. En: Casan C, García RF, Gea GJJ, editores. Fisiología y biología respiratoria. ISB 978-84-8473-572-4. España: Ergon 2007. p. 313-328.
60. Pérez-Padilla R, Torre-Bouscoulet L, Gochicoa-Rangel L, VázquezGarcía JC. 20 años de entrenamiento en espirometría en América Latina. Neumol Cir Torax. 2017;76(1):4-6.

61. Vázquez García JC, Ortiz-Siordia R, Franco-Marina F, SalasHernández, Benítez-Pérez RE, Pérez-Padilla R. Impacto de un curso taller de cinco horas en la interpretación de la espirometría. Neumol Cir Torax. 2019;78(3):270-276.

62. Sandoval-Gutiérrez JL, Monraz-Pérez S, Benítez-Pérez RE, Mireles Cabodevila $E$. Utilidad de la telemedicina en las enfermedades respiratorias. Neumol Cir Torax. 2020;79(1):12-16.

Conflicto de intereses: Los autores declaran no tener conflicto de intereses. 